\title{
What kind of mood influences what kind of memory: The role of arousal and information structure
}

\author{
KLAUS FIEDLER \\ University of Giessen, Giessen, West Germany \\ and \\ WALTER STROEHM \\ University of Marburg, Marburg, West Germany
}

\begin{abstract}
Mood-congruent memory was investigated as a function of the structure of the information to be remembered (categorical vs. isolated) and the participant's arousal (stimulating drug vs. placebo). Free recall of photographs showing either pleasant or unpleasant scenes was assessed for subjects under positive or neutral mood states, induced by the imagination of emotional experiences under relaxation. Although the arousal manipulation did not affect the recall performance, the findings highlight the importance of the information structure: a mood-congruency effect was obtained for the isolated pictures; however, the effect did not appear for categorical material, suggesting that structural constraints on the recall process can override the influence of mood on memory. Interestingly, the subject's evaluations of the pictures also shifted toward their mood states. A general recall advantage of positive mood was observed.
\end{abstract}

Psychological research on the influence of mood on memory (Bower, 1981; Isen, Shalker, M. Clark, \& Karp, 1978; Snyder \& White, 1982) deals with phenomena which appear intuitively highly appealing but for which the empirical evidence is remarkably weak. It is plausible indeed to find that happy people are more prone to remember happy events than sad events (Bower \& Gilligan, 1979) and even more plausible to find that depressive people suffer from selectively pessimistic memories (Beck, 1967; Lloyd \& Lishman, 1975). However, such mood-congruency effects are not consistently obtained in experimentally controlled situations (see Bower, Gilligan, \& Monteiro, 1981; D. M. Clark, Teasdale, Broadbent, \& Martin, 1983). Likewise, it seems plausible to learn that the same information is recalled better when the mood state during the recall test matches the encoding mood state (Bower, Monteiro, \& Gilligan, 1978; M. S. Clark, Milberg, \& Ross, 1983; Eich, Weingartner, Stillman, \& Gillin, 1975). However, such demonstrations of mood-state dependency have been very difficult to produce, and the number of negative results outweighs the supporting cases (see Eich, 1980). Instead of taking the pessimistic view that research on mood and memory is too difficult to yield reliable results, we can also try to resolve the puzzling

The research presented in this article was supported by a grant from the Deutsche Forschungsgemeinschaft and by Homburg-Chemie, Frankfurt. Special thanks to Joseph Forgas for his helpful comments in preparing the manuscript and to Petra Netter for her pharmacological advice. Requests for reprints should be sent to: K. Fiedler, Fachbereich Psychologie, Universitaet Giessen, Otto-Behaghel-Strasse 10, D-6300 Giessen, West Germany. pattern of evidence by looking for moderator variables that help us to understand why mood effects on memory are sometimes obtained and sometimes not. The present paper is concerned with two such factors for which there is already some indication in the literature: the role of arousal and the structure of the information to be remembered. The first factor concerns the further refinement of the mood aspect within the mood-and-memory issue, whereas the second factor pertains to the memory aspect.

\section{Mood and Arousal}

To introduce the arousal hypothesis, we may refer to the associative network analogy, which states that representations of mood states are stored in the same memory system as are semantic concepts and the representations of stimulus properties. Mood influences on memory reflect the associative strength or the proximity between a mood and other memory contents. Thus, evaluatively positive concepts, such as success, pleasure, or beauty, should be associated through past experiences with positive mood states and should be dissociated from aversive mood states, which in turn should be connected with evaluatively negative concepts, such as failure, pain, or ugliness. During a happy mood state, the happy mood node is activated, and part of the activation spreads to the associated nodes, thus raising the probability that moodcongruent material will be accessible for recall.

It seems somewhat strange, of course, to conceive of mood states as nodes in a semantic network (see Simon, 1982), but the idea should not be misunderstood. Only some representations of moods, rather than the mood 
states themselves, are assumed to be located in semantic memory. What exactly makes up the memory representation of mood? A hypothetical answer put foward by M. S. Clark et al. (1983) states that changes in autonomic arousal that accompany moods may be of crucial importance. In one study, for example, they demonstrated an improvement of recall performance when the degree of arousal at the time of retrieval matched the degree of arousal at the time of learning, even when the arousal was produced by radically different procedures (stepping up and down on a cinder block or watching a pornographic film). In another demonstration, these authors found positive attitude statements to be more available when a positive mood state was induced, but this finding was confined to the high-arousal condition. Similar results were obtained by Bartlett and Santrock (1979) who used children as participants and showed that mood influences on memory disappeared when a relaxation treatment prevented autonomic arousal. Bower's (1981) intensity principle, according to which the impact of mood cues on the recall process depends on the intensity of the mood state, is also consistent with the arousal hypothesis.

It is clear that strong arousal cues-like any distinctive context cues-can serve the role of retrieval cues. Despite this evidence, one should be cautious when generalizing the arousal hypothesis. It seems plausible, indeed, that personally involving life experiences (see Bower \& Gilligan, 1979) are linked with arousal cues and, therefore, a happy episode, for example, comes easier to mind under positive mood and particularly when the positive mood state is accompanied by an appropriate degree of arousal. Such an account, however, is hardly applicable when the material to be remembered is neither personally involving nor arousal-producing (e.g., the evaluatively positive or negative words used by Isen et al., 1978). Thus, the contribution of autonomic arousal may turn out to be an exceptional case rather than a necessary condition for the occurrence of mood effects on memory. Moreover, autonomic arousal patterns are of little value for discriminating between qualitatively different moods; their variation within the same mood may exceed the between-mood variance. Part of these difficulties can be overcome if one replaces autonomic arousal by central arousal, which incorporates bodily changes as well as mental changes. It should be noted that such a central arousal hypothesis does not preclude autonomic arousal effects, but only constitutes a less restrictive, more flexible formulation of the arousal principle.

In our attempt to find an adequate operationalization of arousal in the experiment to be reported, we focused on central arousal using a pharmacological treatment. We chose the drug theophyllin (trade name: Captagon; 7-[2( $\alpha$-methyl-phenethyl-amino)-ethyl]-theophyllin $\cdot \mathrm{HCl}$ ). Theophyllin is an amphetamine which causes the production of noradrenaline and adrenaline. The central stimulation is mediated by an increase of the dopamine concentration in the cortex. People who take the drug become less tired, perform better on simple tasks, and report a refreshed state of mind. Certain physiological variables, such as blood pressure and heart rate, may also show a slight increase.

Such a manipulation could provide a pure arousal treatment, in contrast to the treatment by M. S. Clark et al. (1983), which might be confounded with affective properties (e.g., a very dull task in the low-arousal condition or pornographic films in the high-arousal condition) or that might interact with the method of mood induction. For example, it is possible that certain positive mood treatments (e.g., the success feedback provided by M. S. Clark et al., 1983) are reinforced by motor arousal (e.g., produced by stepping up and down on a cinder block), whereas other mood-induction procedures (e.g., imagining a pleasant event) might gain from a relaxed, nonaroused state.

\section{Structure of the Information}

The second variable of interest here, structure of information, causes less conceptual difficulty, and its impact on mood-and-memory phenomena seems easier to predict. To the exent that the material to be remembered can be structured-by category, by serial, by scripts, or by some other kind of principle-the structural redundancy can be utilized to encode the information more economically, to form a memory representation out of higher order units or chunks. Recalling a small number of such higher order units may suffice to reproduce a multitude of specific information that is linked to those units by structural knowledge. For example, remembering that the stimulus list included several color names and several brands of cars can help one recall a dozen specific colors and brands. The recall advantage of structured information is based on a highly systematic retrieval process. Recall protocols are characterized by regular clustering (Bousfield, 1953); primacy or recency effects and other reflections of extraneous factors vanish (Cohen, 1966); and the nature of errors reveals the systematic memory search (Herrmann, Frisina, \& Conti, 1978). The recall process is forced to proceed in clear-cut structural paths, probably bypassing the relatively weak mood cues which provide a rather inefficient alternative for finding the same information in memory. Our prediction, therefore, is that mood influences on memory will be reduced or disappear altogether, to the extent that the recall process is guided by structural knowledge (for a conceptually similar prediction, see Fisher \& Cuervo, 1983).

There is already some evidence to support this consideration. Eich et al. (1975) used categorized word lists and showed that state-dependency effects of marijuana were confined to the categorical level (i.e., more categories were recalled when the retrieval state matched the encoding state) but did not extend to the recall of specific items within categories. Assuming that recall within categories is subject to more structural constraints than is access to the superordinate categories, this finding is consistent with the above prediction. However, Eich et al. did not directly vary the structure of information, and it is not clear 
whether marijuana affords a proper mood manipulation. Moreover, their finding refers to state dependency, whereas we are primarily interested in the phenomenon of mood congruency.

More direct evidence comes from Fiedler, Pampe, and Scherf (in press) using categorically organized behavior descriptions as stimuli. The target person showed predominantly positive behaviors in some categories and mostly negative behaviors in others. No mood-congruency effect (i.e, selective recall of positive behavior under positive mood) was obtained for this redundant body of information. However, there was one exceptional instance in each category, when the target person deviated from the usual pattern (i.e., one positive behavior in otherwise negative categories, and vice versa). In fact, mood congruency was observed for this part of the information, which did not fit the structural constraints. Unfortunately, this finding relies on a data base too meager to warrant a final conclusion (i.e., only one deviating item in each of six categories), and its reliability, therefore, needs to be ascertained in a more suitable test.

The design of the present experiment focuses on the two variables, arousal and structure. Mood and arousal were varied orthogonally as between-subject factors, and the structure of information was varied as a within-subjects factor. The stimulus material consisted of photographs showing either pleasant or unpleasant scenes. Part of the material was organized by thematic categories (e.g., several pictures showing accidents), whereas the rest consisted of isolated pictures that did not lend themselves to categorical coding (e.g., a single picture representing a slaughter scene). According to the above reasoning, any mood-congruency effect was expected to be confined to the unstructured information. Furthermore, the premise here is that memory for pictures is subject to the same mood-congruency effect that has been demonstrated for verbal material. Although this issue is hardly addressed in the literature, the possibility should be considered that the analogous and often idiosyncratic ways of representing pictures in memory, as opposed to the consensual meanings and connotations of the linguistic code, might be insensitive to the operation of mood cues. One subsidiary purpose of the present research is to extend the principle of mood congruency to the domain of pictorial information.

We used an autosuggestive mood-manipulation technique that has also been successfully used in several experiments (Fiedler \& Drewes, 1985; Fiedler \& Fladung, 1986): Mood was induced in a state of deep relaxation in which participants were presumably very sensitive to imaginative thought. Depending on the mood condition, subjects were instructed either to imagine a very pleasant episode from their own lives or to engage in a comparable mental activity that was assumed to be emotionally neutral. We confined ourselves to the manipulation of positive versus neutral mood (as did M. S. Clark et al., 1983 ) and did not include a negative mood condition, as was usually done (e.g., Bower, 1981; Isen et al., 1978). Assuming that not every experiment must include all levels of mood and that others have already compared positive and negative mood, we felt justified to dismiss the negative mood condition if only for ethical reasons. In any case, the relative impact of positive versus neutral mood should be the same, theoretically, as that expected for positive versus negative mood.

\section{METHOD}

\section{Participants}

A sample of 48 male and female students, recruited through several advertisements at the University of Giessen, took part in the experiment. Due to the experimenter's recording error, the data from the first 12 participants ( 3 from each experimental group) could not be analyzed, leaving 36 participants in the final analysis. Students of psychology were excluded from participation because of their experience with similar experiments. The subjects were paid DM 15 for the single session which lasted about $1 \mathrm{~h}$. This relatively high payment is common for experiments in which the participants know in advance that a pharmacological treatment is involved. Subjects were scheduled individually and were allocated to the four experimental groups by turns.

\section{Design}

The joint manipulation of two between-subjects factors (mood and arousal) and two within-subjects factors (stimulus structure and stimulus valence) results in a $2 \times 2 \times 2 \times 2$ factorial design with 9 participants for each combination of the first two factors. Each consecutive subset of 4 participants, one from each condition, saw exactly the same stimulus series as described below.

\section{Materials}

The photographs used as stimulus material were selected in a preliminary study in which 10 judges rated and sorted a total of 48 pictures taken from pictorials, journals, landscape books, and our own archives. The pictures had been originally selected according to the following criteria: (1) their valence had to be classifiable as positive or negative, and (2) each picture either should be a member of some thematic category that also comprised several other pictures or should be a candidate for an isolated instance (i.e., a single-picture category). First, the judges were asked to sort the pictures into as many piles as they wished; they were told, however, that there could be some pictures which do not lend themselves to classification and, therefore, have to be sorted separately. Second, they were presented the pictures once more and had to rate them for valence. The final picture set was based both on the judges' consensus and on the requirement that the categories and singular items be maximally distinctive. The resulting stimulus set consisted of four positive four-item categories (comedians, fun and joy, beautiful landscapes, nice children), four negative four-item categories (diseases, accidents, environmental pollution, threatening technology), eight positive isolated items (e.g., kissing mouth, beautiful sunset, man enjoying his beer), and eight negative isolated items (e.g., violence against woman, slaughtering of seals, burial scene). Thus, the total stimulus series included 48 pictures that were ordered randomly, with the restriction that the distance with which instances from the same category appeared should be maximized. This was accomplished by randomly drawing, without replacement, one picture from each category (in a fixed order) before the second, third, and forth picture per category was drawn. The isolated pictures were interspersed randomly between those drawn from categories. Four consecutive participants, one from each of the experimental groups, always saw the same series in the same order, but the individuals within the groups always received different orderings.

Although the consensus rates on which the stimulus selection was based were rather high (typically about $\mathbf{8 0 \%}$ ), there was always some remaining interpersonal variation, not only in the sorting task, 
which would have been expected, but also in the evaluative judgments. Even when a certain picture appeared to be unambiguously positive or negative, some deviant reactions were given by at least some judges. Therefore, we decided to reassess the two relevant properties of the stimuli (valence and clustering) for the participants in the main investigation (see below).

\section{Procedure}

Participants were welcomed in a friendly manner and were given time (filled with small talk) to accommodate to the experimental situation. They were told that the experiment would be concerned with the influence of viewing slides on an imagery task. However, they were not informed that they would be given a recall test. In an anticipatory overview of the following hour or so, the three most salient aspects of the experimental situation were outlined: the drug, the series of slides, and the imagery task to be performed under relaxation. The drug was (correctly) described as a substance which is used in order to increase mental performance and concentration, thus justifying its use as an integral part of the imagery task. Although the commercial name (Captagon) of the substance was not mentioned, the participants were thoroughly informed about all the possible side effects and counterindications. After the participant had confirmed his or her decision to participate, he or she was presented a glass of water along with the drug which either contained $50 \mathrm{mg}$ theophyllin or was a placebo. The possibility of a placebo was never mentioned, and the experimenter was blind with respect to the subject's drug condition.

Immediately after the intake of the drug, the room was darkened and the series of slides was presented at a constant rate of $15 \mathrm{sec}$ per slide. The size of the resulting images on the projection screen, which was placed at a distance of $2 \mathrm{~m}$ from the participant, was about $120 \times 80 \mathrm{~cm}$. In the experimenter's brief instructions, the participant was asked to consider the slides thoroughly and was encouraged not to suppress his or her affective reactions to the photographs.

The stimulus presentation was followed by a $30-\mathrm{min}$ period of irrelevant activity that consisted of an informal but well-structured interview about the participant's age, professional goals, consumption of coffee or other stimulating agents, study interests, and other everyday issues. Mood-relevant topics were avoided carefully. At the end of the interview, each participant was also asked to rate his or her mood at the time of first entering the experimental room. (Since this measure did not differ between groups, it will not be mentioned again.) The intention was mainly to establish a delay between the intake of the drug and the stimulus presentation on the one hand and the recall test on the other hand. There are two reasons for this delay: first, to produce the maximal effect of the pharmacological treatment, which is reached about $40 \mathrm{~min}$ after intake, and second, to ascertain that the stimulus encoding process was virtually over when the following mood manipulation began, which should only affect the retrieval mood.

Sitting in a comfortable reclining chair, the participant first performed a relaxation procedure, which was essentially modeled after Jacobson's (1938) "progressive relaxation." Prior experience with this procedure had shown that even untrained participants usually reach a state of considerable relaxation within a few minutes. After subjects reached this relaxation state, the mood manipulation began. Participants of the positive mood condition were asked to think of a very pleasant episode in their own lives and to try to experience the same emotional quality that accompanied the original event. Having accomplished this, the experience was intensified and elaborated via imagery and attempts to revive the original sensations, perceptions, and affective reactions. Participants were encouraged to conserve for a while the emotional states they had reached. They were instructed not to tell the experimenter about the kind of experience they imagined, nor to reveal anything about their private fantasies. Obviously, this procedure highly resembles the hypnotic treatment used by Bower and colleagues. When the subject was in the neutral mood condition, the imagination of a pleasant episode was replaced by another imagery task selected to be emotionally neutral but to represent roughly the same level of cognitive effort. The participant had to imagine a map of the European continent and to assess the geographic phenomena appearing before his or her mental eye.

We decided to avoid a distracting and possibly demand-producing manipulation check at this point. Instead, an independent sample of 20 subjects were treated in the same way, 10 receiving the positive mood manipulation and 10 the neutral mood manipulation. The effectiveness of the treatment was confirmed. While many bodyrelated and arousal-related questions revealed no difference, the positive mood condition did produce significantly more positive descriptions of one's own feelings, as well as more positive associations to 20 ambiguous words and to 11 ambiguous pictures, than did the negative mood condition. Since similar results were obtained in two other experiments (Fiedler \& Drewes, 1985; Fiedler \& Fladung, 1986), the effectiveness of the mood manipulation can be taken as established.

The free recall test was administered while the participants remained in the comfortable reclining chair. They were simply asked to recall, in any order, as many pictures as possible by identifying the pictures with appropriate key words. No time limit was set. The phrase "recall test" was avoided because we did not want the task to resemble an achievement test. Instead, the test was introduced as an appraisal of what makes up the participant's consciousness at that moment. The recall test responses were given orally and were tape-recorded.

After the participant felt that his or her memory was exhausted, the experimenter led him or her to another part of the room where the sorting task had been prepared. As in the preliminary study, the 48 pictures had to be sorted into thematic piles and the possibility to establish single-picture piles was mentioned. Finally, the same pictures were presented once more and the participant had to categorize each stimulus item with regard to its evaluation as either positive, neutral, or negative. This procedure was employed to double-check the category structure and valence data already obtained in the pilot study. Before they left the laboratory, the participants were questioned about the relaxation technique, the imagery task, and the drug. They were handed a closed envelope containing DM 15 and an indication of their drug condition (placebo or Captagon).

\section{RESULTS AND DISCUSSION}

\section{Mood and Recall}

A $2 \times 2 \times 2 \times 2(\operatorname{mood} \times$ drug $\times$ valence $\times$ structure $)$ factorial analysis of variance was performed on the free recall proportions computed for each participant and within participants for categorically structured positive items, categorically structured negative items, singular positive items, and singular negative items. The ANOVA results are based on an arcsine transformation of the empirical proportions, but the reported means have been retransformed to represent the natural proportion measure. (It should be noted, parenthetically, that the results are essentially unaffected by these transformation operations.)

Since the individual participant's subjective evaluations sometimes differed from the categorization of positive and negative stimuli in the pretest, we conducted separate ANOVAs with the stimulus valence factor based on both kinds of classification. The influence of experimental conditions on the recall performance are summarized in 
CATEGORIZED PICTURES

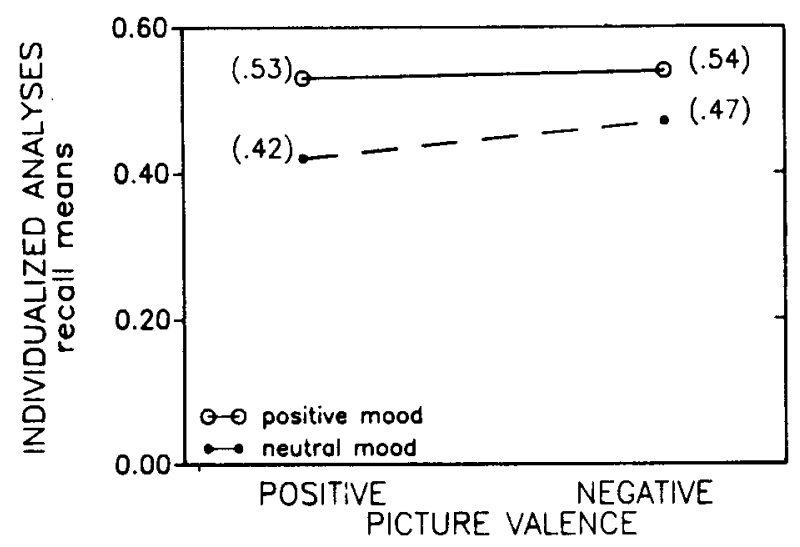

CATEGORIZED PICTURES

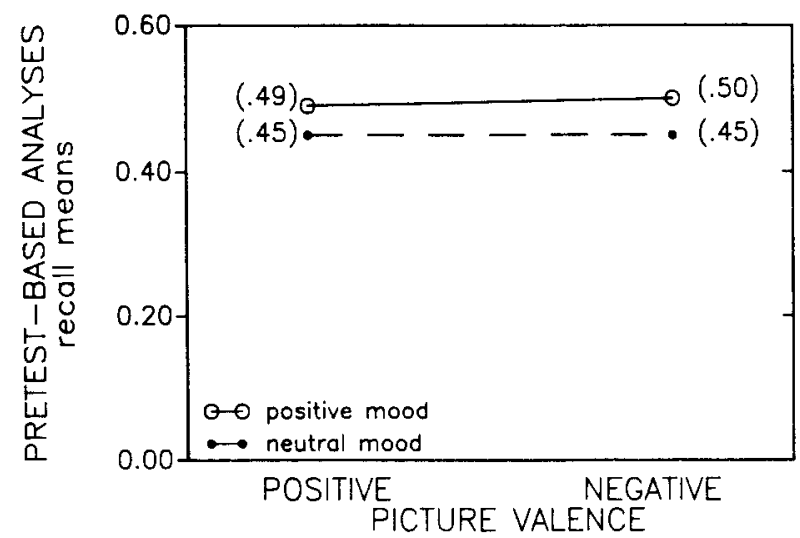

ISOLATED PICTURES
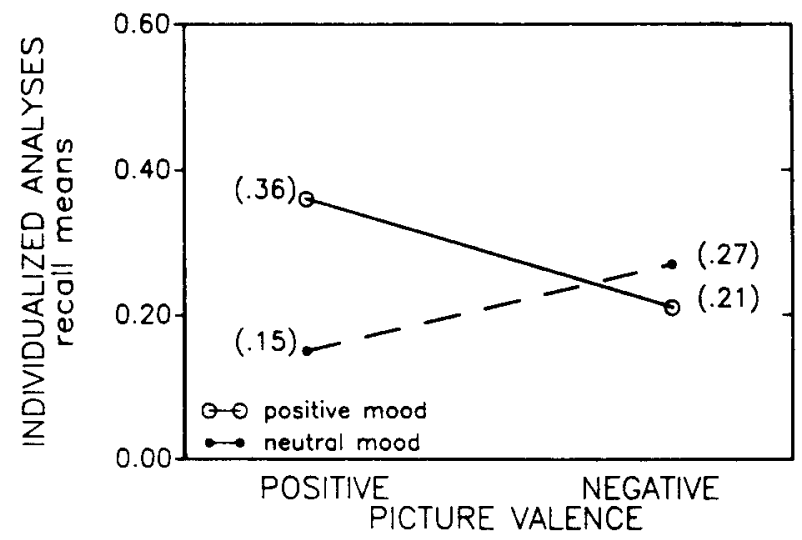

ISOLATED PICTURES

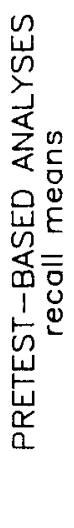

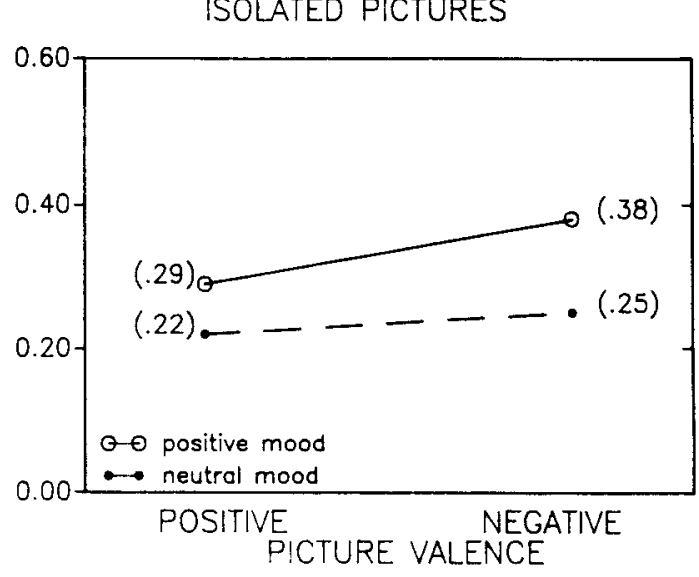

Figure 1. Mean proportion of pleasant and unpleasant pictures recalled by participants in the positive and neutral mood conditions, split for isolated and categorized pictures. The two graphs on top are based on the individualized assessment of picture valence, and the two lower graphs refer to the pretest-based analysis.

Figure 1, where the upper two graphs present the individualized data and the lower two graphs present the pretest-based data.

Although it is evident from both analyses that categorical information is recalled much better than is isolated information [individualized: $F(1,32)=43.76, p<.001$; pretest: $F(1,32)=27.60, p<.001]$ and that the positive mood group was superior to the neutral mood group [individualized: $F(1,32)=4.37, p<.05$; pretest: $F(1,32)=4.48, p<.05]$, the expected pattern of interactions only emerged from the individualized data. Thus, the analysis which takes the individual stimulus evaluations into account is necessary to demonstrate the mediating role of information structure for mood congruency. As predicted, the upper graphs of Figure 1 reveal a stimulus valence $\times$ mood interaction $[F(1,32)=6.65, p<$ $.05]$, indicating the advantage of mood-congruent material. This finding, however, is completely due to the isolated pictures and disappears for the categorical pictures, as reflected in a significant stimulus valence $\times$ stimulus structure $\times$ mood interaction $[F(1,32)=5.12$, $p<.05]$. In accordance with our assumptions, then, pleasant pictures were recalled better than were unpleasant ones under positive mood, and recall of unpleasant pictures was superior to that of pleasant ones under neutral mood, provided there are no structural constraints that suppress such a mood-congruency effect. The corresponding interactions for the pretest-based data are not significant $[F(1,32)=0.42$, and $F(1,32)=0.25$, respectively $]$ No other main effect or interaction, especially none involving the arousal factor, reached or approached statistical significance.

On the one hand, it seems plausible that interactions in a four-factorial design can be demonstrated more reliably when interpersonal differences are taken into account (the individualized analysis). On the other hand, however, the different outcome of the two analyses raises some interpretational problems which need to be discussed briefly.

First, the possibility can be dismissed that differences in the degree of positive and negative valence between isolated and categorical pictures became apparent only in the individualized analysis, and this might account for the reported findings. Thus, isolated pictures might have been judged to be more unequivocally positive or negative, rendering a congruency effect more likely. Inspection of the data, however, shows that, if anything, the reverse is true. 
Second, it should be added that if the influence of structure were due to any other confounded difference in the objective contents of isolated and categorical pictures, the results should have been more pronounced when based on the invariant pretest classification.

Third, another interesting consideration stems from previous research by Nuttin (reported in Nuttin \& Greenwald, 1968) showing that successful recall of an item increases its judged positivity. This influence might have biased the individual stimulus evaluations at the end of the experiment. However, regression analyses with pretest valence and recall success as predictors and subjective valence as criterion, conducted for each individual judge, yielded a median beta of 0.80 for pretest valence but a median beta of exactly zero for recall success.

\section{Mood and Stimulus Evaluations}

One question that arises is whether the evaluative reactions to the pictures at the end of the experimental session were also affected by the mood manipulation. Scoring $+1,0$, and -1 , respectively, for pictures categorized as positive, neutral, and negative, a mood $\times$ drug $\times$ valence analysis of variance with pictures as the unit of analysis revealed that this was actually the case. The same pictures were judged significantly more positive by positive mood participants (mean $=+.59$ and -.78 for pleasant and unpleasant pictures, respectively) than by neutral mood participants [mean $=+.47$ and -.83 ; $F(1,46)=11.33, p<.005]$. Thus, the meaning and the affective connotations of the stimuli themselves were assimilated to the participant's mood state. The important methodological implication of this finding is that perceived stimulus valence and mood may be impossible to manipulate independently. We shall return to this point later.

However, in the present case, the mood-dependent evaluation shift does not render the mood-congruency effect artificial, because the valence ratings were in fact assessed after the recall test was already finished. That is, if the shift was produced artificially by the judgment task, it cannot have affected the recall test. If, however, the evaluation shift occurred during the recall process, then it was not created artificially and must be regarded an integral part of the cognitive mechanisms that lead to mood congruency. Unfortunately, we are not in the position to decide whether the induced mood state really persisted until the recall test was over and affected the evaluative judgments or whether the evaluation shift reflects an influence of the preceding recall task on the subsequent stimulus evaluations. This raises the interesting hypothesis that the evaluation shift may constitute a necessary aspect of the memory process itself. If we had administered a manipulation check at several points in the procedure, we could say more. Such repeated manipulation checks, however, are often problematic because of demand characteristics and self-consistency effects.

A significant mood $\times$ drug interaction $[F(1,46)=7.64$, $p<.01]$ indicates that the mood influence on the evaluative reactions was more pronounced in the placebo con- dition than in the Captagon condition. This interaction is virtually confined to positive pictures so that the threeway interaction is also significant $[F(1,46)=5.47, p<$ $.05]$. As displayed in Figure 2, positive pictures were seen in a positive light by Captagon subjects, regardless of their mood condition, whereas placebo subjects tended to judge the same pictures as less positive under neutral than under positive mood. Assuming the reliability of this finding, it means that either good mood or Captagon is sufficient to increase the positive reactions to the slides.

Of course, the main effect for the valence factor was also highly significant $[F(1,46)=406.08, p<.001]$, indicating that positive and negative pictures were clearly judged as different. Thus, despite the interpersonal variation in the judges' evaluative reactions, the manipulation of the stimulus valence was undoubtedly successful. The same conclusion can be drawn from the analysis of the clustering data, supporting the manipulation of the stimulus structure. Most judges sorted the pictures into the appropriate categories, and the typical consensus rate was 30 or 40 out of the 48 judges. Moreover, the isolated items were rarely classified into one of those
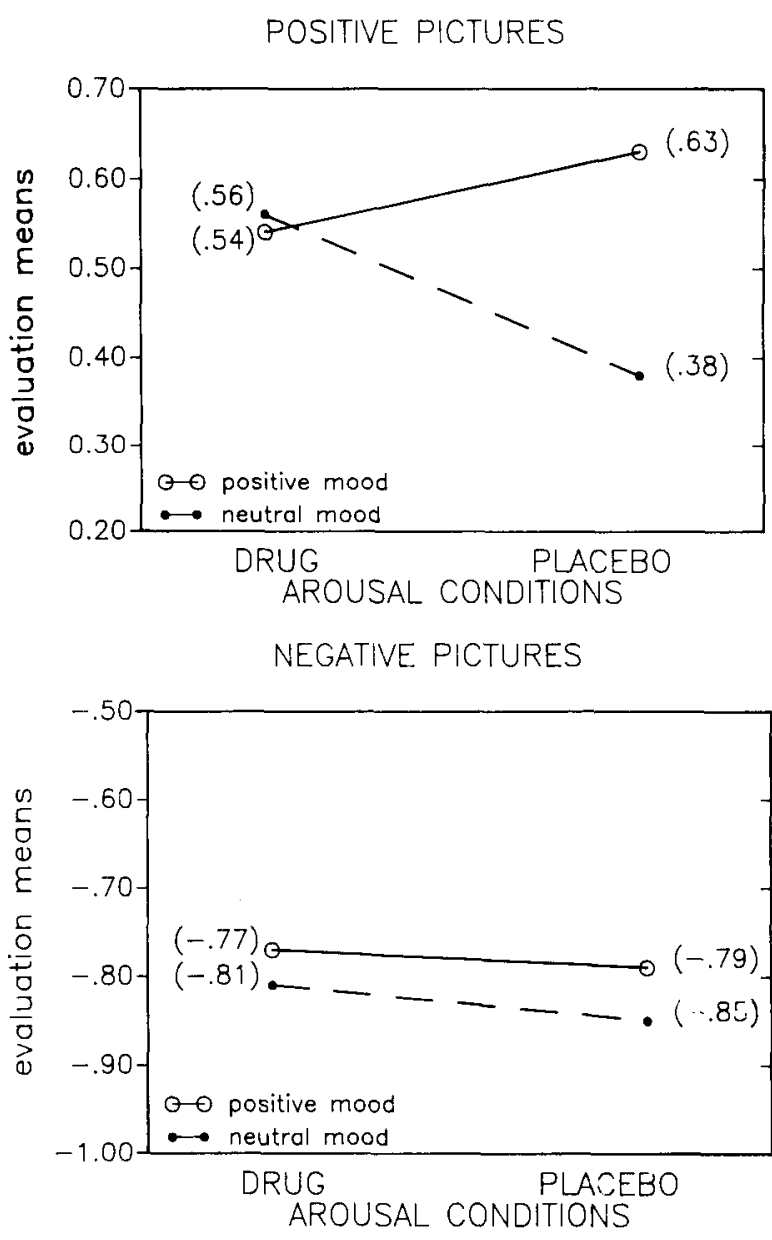

Figure 2. Mean evaluative judgments of pleasant and unpleasant pictures by participants in different mood conditions and drug conditions. 
categories. In one word, the construction of the stimulus material seems to have been as satisfactory as could be expected, and the residual variation between individuals should be regarded as an inevitable matter of fact.

To summarize the present results, it has been shown that the phenomenon of mood congruency can also be obtained with pictorial material and when only the participants' mood at the time of retrieval is manipulated. Apart from a general recall advantage in the positive mood condition, relatively more pleasant pictures were recalled under good mood while relatively more aversive pictures were recalled under neutral mood. As expected, this congruency effect was confined to the unstructured part of the stimulus material and was absent for the categorically organized pictures. Thus, our predictions regarding the moderating influence of information structure on moodand-memory phenomena are confirmed.

Theoretically, the categorical structure of the information leads to a recall process proceeding in two stages: (1) access to the superordinate categories on which the memory representation is based, and (2) reconstruction of specific items within categories (see Bahrick, 1971; Cohen, 1966; Eich et al., 1975). That is, the role of the category meanings is comparable to the role of the cues in a cued recall test, and it is interesting to note that mood effects which are present in free recall have been reported to be absent in cued recall (Eich et al., 1975), presumably because the associative links between the cues (categories) and the stimuli to be remembered are much stronger than the relatively weak links between mood states and stimuli. For similar reasons, mood effects have not been found in a recognition test (Bower \& Cohen, 1982 ), which is the most radical form of a cued recall test, in that the stimulus itself is presented as a cue. The present finding is quite consistent with this evidence, and all of these results are not difficult to interpret within the associative network analogy.

Our second hypothesis regarding the role of arousal in mediating mood effects on memory did not receive support. The abnormally low level of somatic and autonomic arousal that was induced by the deep relaxation procedure did not prevent the occurrence of mood congruency, and the intake of a centrally stimulating drug also failed to affect the results. One might argue that this negative finding concerning the arousal hypothesis is outweighed by the positive findings reported by $\mathrm{M}$. S. Clark et al. (1983) and Bartlett and Santrock (1979). Of course, nobody would seriously doubt the possibility that arousal can potentially reinforce the psychological influence of mood states or that arousal cues can enrich the representation of encoded information. However, assuming the reliability of the present results, we feel justified to conclude that arousal must not be considered a necessary precondition for mood effects to occur, as could be inferred prematurely from the evidence cited above. Although certain mood treatments (e.g., success feedback) may be strengthened by arousal, it is also conceivable that other treatments (e.g., imagining pleasant events, as in our case) gain from relaxation.

Parenthetically, it should be mentioned that the pharmacological treatment was not completely mute. Apart from the drug $\times$ mood interaction for the picture evaluations already mentioned, another drug $\times$ mood interaction $[F=9.05, p<.01]$ was obtained in a subsidiary ANOVA for the amount of clustering, based on the clustering index proposed by Roenker, Thompson, and Brown (1971). An earlier finding (Fiedler et al., in press) that the systematic clustering of items according to categories is reduced under positive mood (suggesting a less systematic recall strategy; see Isen, Means, Patrick, \& Nowicki, 1982) could only be replicated for the placebo condition, whereas an opposite tendency was observed in the drug condition. Although we make no attempt to provide post hoc explanations for these unexpected findings, they nevertheless suggest that something was caused by the pharmacological agent.

\section{CONCLUSION}

An intriguing problem is raised by the demonstration that the affective contents of the stimuli themselves are not independent of the participant's mood state. From a traditional perspective, this may be considered as indicating a methodological difficulty, because experimental research starts from the premise that mood states and stimulus properties can be varied independently. If the meaning and the experience of the stimuli are not the same for subjects of different mood conditions, the independence assumption must be given up and the power of the experimental method is at least partially lost. From an alternative perspective, however, we could also recognize that it is never the objective stimulus that comes to interact with the individual's independent mood state. What we perceive and encode in memory is not the stimulus itself but, rather, our reactions to and experience of that stimulus. The implication is that the psychologically effective stimulus always represents some kind of fusion of stimulus characteristics on the one hand and characteristics of the individual on the other hand. Thus, each pleasant stimulus can be regarded as a minor positive mood treatment that will interfere a bit with a negative mood state and slightly reinforce a positive mood state, whereas each unpleasant stimulus may represent a minor negative mood treatment. According to this view, stimuli and mood treatments would be treated as theoretically equivalent except for their intensity and the temporal extension of their effect. The individual's more enduring mood state would then be regarded as a kind of anchor, and the tendency to selectively encode or retrieve congruent stimuli could be regarded as assimilation processes with respect to that anchor. Within such a conceptual frame, it would be interesting to ask whether contrast effects (e.g., cases of incongruence) will also be obtained under some conditions (as already evident in Fiedler et al., 
in press). In any case, however, it is an intriguing task for future research to clarify the mutual interaction of the individual's mood and the affective qualities of the stimulus.

\section{REFERENCES}

BAHRICK, H. P. (1971). Accessibility and availability of retrieval cues in the retention of categorized lists. Journal of the Experimental Psychology, 89, 117-125.

Bartlett, J. C., \& Santrock, J. W. (1979). Affect-dependent episodic memory in young children. Child Development, 50, 513-518

BECK, A. T. (1967). Depression: Clinical, experimental, and theoretical aspects. New York: Harper \& Row.

BousfiELD, W. A. (1953). The occurrence of clustering in the recall of randomly arranged associates. The Journal of General Psychology, 49, 229-240.

Bower, G. H. (1981). Mood and memory. American Psychologist, 36 129-148.

Bower, G. H., \& CoHEN, P. R. (1982). Emotional influences in memory and thinking: Data and theory. In M. S. Clark \& S. T. Fiske (Eds.), Affect and cognition. Hillsdale, NJ: Erlbaum.

Bower, G. H., \& Gilligan, S. (1979). Remembering information related to one's self. Journal of Research in Personality, 13, 420-432.

Bower, G. H., Gilligan, S. G., \& Monteiro, K. P. (1981). Selectivity of learning caused by affective states. Journal of Experimental Psychology: General, 110, 451-473.

Bower, G. H., Monteiro, K. P., \& Gilligan, S. G. (1978). Emotional mood as a context for learning and recall. Journal of Verbal Learning \& Verbal Behavior, 17, 573-585.

Clark, D. M., Teasdale, J. D., Broadbent, D. E., \& Martin, M (1983). Effect of mood on lexical decisions. Bulletin of the Psychonomic Society, 21, 175-178.

Clark, M. S., Milberg, S., \& Ross, J. (1983). Arousal cues material stored in memory with a similar level of arousal: Implications for understanding the effects of mood on memory. Journal of Verbal Learning \& Verbal Behavior, 22, 633-649.

COHEN, B. H. (1966). Some-or-none characteristics of coding behavior. Journal of Verbal Learning \& Verbal Behavior, 5, 182-187.

EICH, J. E. (1980). The cue-dependent nature of state-dependent retrieval. Memory \& Cognition, 8, 157-173.

Eich, J. E., Weingartner, H., Stillman, R. C., \& Gillin, J. C.
(1975). State-dependent accessibility of retrieval cues in the retention of categorized lists. Journal of Verbal Learning \& Verbal Behavior, 14, 408-471.

FIEDLER, K., \& DREWES, R. (1985). Retrieval mood effects on recall following different encoding tasks. Unpublished data, University of Giessen, Giessen, West Germany.

Fiedler, K., \& FLADUNG, U. (1986). Mood congruence in productive and reproductive memory. Unpublished manuscript, University of Giessen, Giessen, West Germany.

Fiedler, K., Pampe, H., \& Sherf, U. (in press). Mood and memory for tightly organized social information. European Journal of Social Psychology.

Fisher, R. P., \& Cuervo, A. (1983). Memory for physical features of discourse as a function of their relevance. Journal of Experimental Psychology: Learning, Memory, \& Cognition, 9, 130-138.

Herrmann, D. J., Frisina, R. D., \& Conti, G. (1978). Categorization and familiarity in recognition involving a well-memorized list. Journal of Experimental Psychology: Human Learning \& Memory, 4, 428-440.

Isen, A. M., Means, B., Patrick, P., \& Nowicki, G. (1982). Some factors influencing decision-making strategy and risk taking. In $\mathbf{M}$. S. Clark \& S.T. Fiske (Eds.), Affect and cognition. Hillsdale, NJ: Erlbaum.

Isen, A. M., Shalker, T. E., Clark, M., \& KarP, L. (1978). Affect, accessibility of material in memory, and behavior: A cognitive loop? Journal of Personality \& Social Psychology, 36, 1-12..

JACOBSON, E. (1938). Progressive relaxation. Chicago: Chicago Press. Lloyd, G. G., \& LiShman, W. A.(1975). Effect of depression on the speed of recall of pleasant and unpleasant experiences. Psychological Medicine, 5, 173-180.

NutTin, J., \& GREenwAld, A. G. (1968). Reward and punishment in human learning. New York: Academic Press.

Roenker, D. L., Thompson, C. P., \& Brown, S. C. (1971). Comparison of measures for the estimation of clustering in free recall. Psychological Bulletin, 76, 45-48.

SIMON, H. A. (1982). Affect and cognition: Comments. In M. S. Clark \& S. T. Fiske (Eds.), Affect and cognition. Hillsdale, NJ: Erlbaum.

SNYDER, M. \& WHITE, P. (1982). Moods and memories: Elation, depression, and the remembering of the events on one's life. Journal of Personality, 50, 149-167.

(Manuscript received April 4, 1985;

revision accepted for publication September 4, 1985.) 